\title{
Business Growth Using Open Source e-Commerce and ERP in Small Business
}

\author{
Valtteri Kujala ${ }^{1}$ and Raija Halonen ${ }^{2}$ \\ ${ }^{2}$ University of Oulu, P.O.Box 4500, 90014 Oulu, Finland \\ ${ }^{1}$ valtteri.kujaladvalfi.fi ${ }^{2}$ raija.halonendoulu.fi
}

\begin{abstract}
The purpose of the current study was to introduce a solution to support small business to maintain its continuity and to grow its business in the competition among other companies. Earlier knowledge reports the role of ERP in managing business and points out ability to improve ERP systems later. Current ICT-enabled tools enable efficient means to communicate with customers and other stakeholders, and to add visibility of the companies. A prototype for an ERP system was built utilizing an open source platform WordPress and its ready-made plugins. Among other basic ERP features, the solution offered tools for web publishing and a simple authentication system. Based on the study, the chosen WordPress infrastructure seemed satisfactory for small business needs.
\end{abstract}

Keywords: Small Business, e-Commerce, ERP, Open Source.

\section{Introduction}

The purpose of the current study was to introduce an efficient solution including essential functionalities to support small business to strengthen its continuity and to grow its business in the competition among other service providers. As the world is more connected than ever before, the opportunity to start electronic business is available globally also for micro size companies.

Small and medium-sized enterprises (SMEs) are often seen as acting similarly in utilizing information technology (IT) despite their differing sizes. However, the organizational and environmental conditions of their potentials to adopt IT differ. [1] Globally, the role of SMEs is ever more seen as the backbone for economic growth. SMEs provide job opportunities; they are significant goods suppliers and service providers also for large enterprises. [2] In this paper, the focus is on small business.

Globally, digitization has emerged as a key driver that speeds growth and encourages job creation. However, the role of digitization by country and by sector is not shared evenly. Countries with developed economy experience high economic growth while others tackle with challenges to see benefits brought by new technology and potentials of digitization. [3]

The current research problem emerged from the comprehension that small business needs to be competent among other actors to survive, and new solutions are needed to spur performance, visibility and connectivity. There are possibilities to grow business in the local country but even more abroad as emerging markets are adopting means of 
mobile platforms (see e.g. [4],[5]). One of the valuable features of enterprise resource planning (ERP) constructions is to identify critical needs in business processes than can be assisted with ERP features and functions [6].

The research problem was solved by answering a research question: "How to build an integrated e-commerce and ERP system for small business?" The solution was built by applying design science research (see [7]). In the build, a prototype of the integrated e-commerce and ERP system with WordPress was constructed, simulated and evaluated. The main contribution of the study was to get a solution to be used in the business of an existing software company, and to get a solution to be further tested in wider environments in other business areas.

The paper continues by presenting earlier related knowledge, followed by describing the actual research design and implementation, and ending by discussion and conclusions.

\section{$2 \quad$ Earlier Studies}

According to a seminal paper of Greiner [8], business growth was seen as a continuum of five phases: creativity, direction, delegation, coordination, and collaboration. While growing, organizations faced stages of crisis such as crisis of leadership, crisis of autonomy, crisis of control and crisis of red tape. An unidentified crisis appears when the organization had achieved the most developed stage (collaboration). Greiner updated his seminal paper in 1998 by adding that the five-phased continuum did not consider knowledge organizations or service businesses that were to grow in future.

Developed and ever developing technology and innovation influence growth of business in several ways. For instance, there are new tools enabled by new ICT to build new services for communicating with customers and service providers, and to add visibility of the enterprises. [9] In this sense, the role of ERP is crucial. In the implementing phase, special attention should be in reviewing the pre-implementation process, installing the right hardware and software and train users of the system. Data accuracy should be also critically highlighted, and the ERP system improved continually after its installation. [6]

Effective governmental actions are needed to produce fruitful infrastructures for new innovative companies to build economic growth. The actions should focus on different kinds of innovative entrepreneurship environments that foster new innovative ideas and companies, and that collaborate with universities to transfer knowledge between education and practice. There is not a single infrastructure that causes growth, but companies should collaborate and combine infrastructures. Furthermore, the policymakers should encourage companies to collaborate with technology centers, incubators and universities, and get benefit entrepreneurship services offered by these institutions. [10]

Due to digital disruption, the landscape of business has become more global and interconnected, leading to increased competition with new rules in the markets [11]. The role of management is challenged by the need to monitor new tools and possibilities to communicate with their partners and stakeholders, not to forget the customers. Likewise, new kind of requirements and regulations related to e.g. environmental opportunities need to be covered by the management. [9] 
Internet-enabled e-commerce seems to develop with increasing pace, and business practitioners need to consider updating their practices to meet new requirements set by the business environment. The processes change, and stakeholders are waiting for prompt responses according to their needs, enabled by means of internet and replacing legacy manual activities. [12] However, there are automatic tools to support or facilitate evaluation remotely [13]. E-commerce platform systems and applications have had a unique impact on global business and sales, incomparable to any other innovative development trend, especially in developing countries [14]. Infrastructure and cost are critical factors in e-commerce growth. For small businesses, a cost-efficient ecommerce platform can be one of the most important tools enabling them to offer their products and services to clients and other businesses to survive in the digital world. [15] If mobile usability is not respected in web design, it can cause significant issues for mobile users leading to frustration and poor customer experience [5]. Furthermore, increased staff awareness and skills in technology are important factors driving motivation to get into e-commerce. What comes to technical infrastructure e-commerce technology, network and internet speed infrastructures are crucial: if the e-commerce platform is difficult to access and maintain, businesses and customers are not seeing it a valuable part of their business. [15]

Current digital technologies are already seen spurring business in several ways like applying social media, mobile, analytics or embedded devices to improve customer experience, business operations or creating new business models [16]. There already are several models describing business life cycle and how new business emerge and grow. However, more research is needed to add knowledge about enterprises in versatile areas and ways that support and spur business. A constructivist model building on carefully chosen characters of earlier presented model and noting proposed stages is expected to influence both business development and entrepreneurship research. [17]

Open source can allow companies to share and implement their information. In the development of the web technologies, the open-source code has been a very powerful force and has helped greatly in preventing excessive power from commercial groups and companies. The transformation towards open source in design progress has also helped to articulate more democratic and borderless design and development work in the world of IT. [18]

The web-based open source platform was used by $30 \%$ of the websites from small business websites to large portal-type web applications used by, i.e., Mercedes-Benz, Bloomberg, and Vogue [19]. WooCommerce is one of the most popular free WordPress plugin to be installed on the platform to extend WordPress with e-commerce features. The high popularity on WordPress has been leading the growth also in WooCommerce, as it integrates quickly and naturally to the platform as a free plugin. [20] WooCommerce was the most popular e-commerce platform and used over two million sites around the world in 2017 surpassing other popular closed platforms such Shopify, Magento Enterprise, and Oracle commerce [21].

Furthermore, societies and communities can expect notable economic benefits and see significant social benefits if they build and foster digitization in their environments. The improvement can be seen in increased productivity, new business, increased employment and improved quality of life in the communities. [3] Mobile electronic commerce and the popularity of the smartphone and public internet infrastructure spending is increasing, especially in emerging markets such as in China and India. [4]. 
A recent study about the role of ICT in micro business in Kongo suggested that policy makers in developing areas should promote mobile device, ICT training and micro entrepreneurship to foster economic development, and that achievements in mobile technology might add micro entrepreneurship to grow in many product branches. [22] The role of SMEs is significant covering $99 \%$ of all business in the EU. [23]

Small business can be referred to business, which is usually independently owned and one of the many similar businesses in the marketplaces. It usually has limited resources to operate and invest [24]. ERP systems are crucial for SMEs to collaborate with bigger companies [25]. A recent literature review analyzed issues related with coordination and responsiveness of SMEs and pointed out how IT applications have been seen as important drivers in coordinating supply chains. SMEs have not adopted supply chain management in their business and are thus facing problems related to supply chains. However, more research is needed to improve several tasks such as coordination and service quality. [2]

Successful e-commerce business is connected to the usability of the system. Google analytics can provide evaluation and metrics that provide information about usability problems in web-based e-commerce systems. Google analytics is cheap to use, and it can be accompanied with other evaluation methods such as heuristic evaluation. The cost of employing the evaluation in e-commerce websites is crucial whether evaluation is done user-based, evaluator-based or software-based, such as Google Analytics. Cheap and quick tools such as Google analytics can provide sufficient information about usability issues. Using Google analytics in evaluation does not require specific test users or questionnaires reducing the cost of usability research. [26]

\section{The Study}

In this section, the construction and development process of the prototype system is explained. Design science research described by Hevner et al. [7] was applied because one of the goals in design science research is that it should lead to a better collaboration and work between the different audiences and parties allowing more different and efficient designing to happen. The research should keep as its main objective creating solutions to relevant business problems.

The system was built using open-source platform WordPress and customized to achieve the desired outcome for prototype. Before starting to develop the system, it was essential to understand what kind of features (plugins) small businesses would need from the system. The needs would vary from business to business. It would be complex for integrated e-commerce and ERP system to handle all diverse functions of both product and service focused businesses. The current prototype was decided to be limited for small businesses producing and selling physical products. The manufactured products would be tracked in production management and then kept in the inventory until sold in the e-commerce via public frontend.

To be utilized in small businesses producing and selling physical goods, basic features of web-based e-commerce and ERP system were determined sufficient for the scope of the study. These features included typical e-commerce product sales, payment methods, customer database, administration, web frontend and contact forms with basic ERP features such as production and inventory management supporting business 
growth. Building the system from using open source also made it possible to add new features to the system later. That also made it easier to optimize resources and costs needed on implementation when it was not necessary to build or activate all features and functions of the ERP all at once. Utilizing open source platform WordPress and its ready-made plugins, it was possible to make the development work rapidly in nine working weeks (Fig. 1).

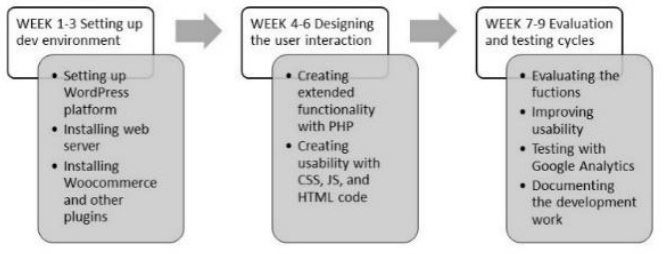

Fig. 1. The implementation plan for the system.
Before the development was started, a plan for the infrastructure was formed to build the prototype (Fig. 1). The estimated schedule also included selecting and studying the WordPress platform, which formed the backbone of the prototype system. The first weeks mostly were used studying the WordPress platform, open source community and testing various plugins followed by the customization done mostly with PHP coding and visualizing the usability with CSS, JS and HTML coding. The evaluation was done when the system was functioning with satisfactory ecommerce and ERP features.

A standalone WordPress installation offered basic features for web publishing, writing articles, pages and posts to be published online, and a simple authentication system with different user levels and rights. For example, the administrator could allow only certain users to publish new posts while the other users could only edit articles without posting them. The authentication levels in the prototype were divided into two user types, the business owner acting as Administrator and the e-commerce customer browsing products and making purchases as a guest or a registered customer (Fig. 2).

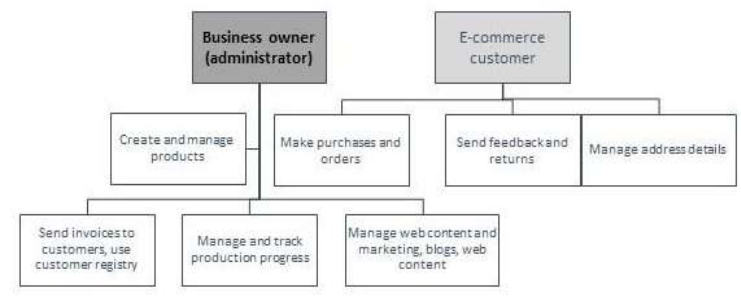

Fig. 2. User levels and privileges. The administrators had all the privileges to update, add, and manage information in the system creating new products, pricing products, managing inventories and productions and, also the authority to create other users. The customers could make purchases, view past orders and invoices, send feedback and returns, and edit their contact and delivery information. The customers had no access to the backend of the system or any controls; they only could make and monitor their own orders and send feedback with product returns and manage their customer information.

Most features for the prototype system were found from WordPress plugin repository and the rest were developed with custom PHP code. WooCommerce ecommerce features could be extended with custom PHP code to support ERP features, such as production management.

The simplest plugin to be utilized in WordPress could be a simple PHP file that could be uploaded to the core file system. When the new script had been uploaded, it could 
be activated in the WordPress settings and functions of that module would become live immediately. In the simplest case, the module could print out "hello world" to every page or product of the system. However, usually, the modules are much more complex and adding advanced functionalities to the platform. Developing plugins have created even an entire industry to the web where plugin developers are selling their developments. As $30 \%$ of the websites were found to be using WordPress (WordPress.org, 2018), it could be assumed that needs for different plugins were enormous. Usually, the modules offered much more functionalities and actions to be added to the base WordPress. One example of the module was the e-commerce platform WooCommerce, which was enabled in the prototype (Fig. 3). The ERP functionalities, such as production management, was developed as separate custom plugin to the platform.

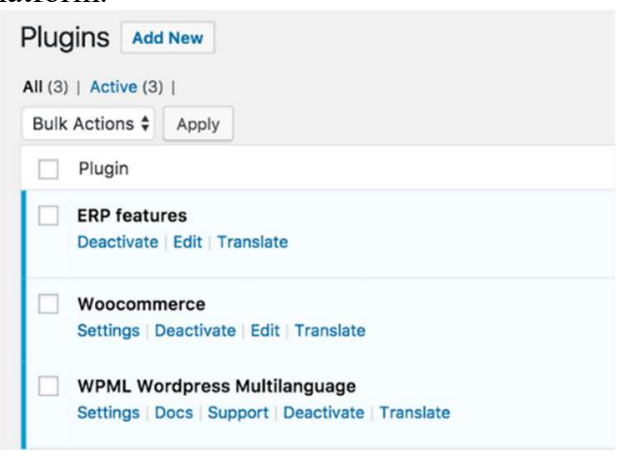

Fig. 3. Managing plugins in the platform.

Adding and managing plugins in WordPress was easy via Plugin management settings. However, adding unnecessary modules could add unwanted complexity and sluggishness to the WordPress system. Every plugin installation and feature in the platform should be a well-educated decision to avoid security threats and issues with reliability and speed. It was purposeful to use only the plugins we needed for the system. It was decided to use only two additional plugins with WordPress, WooCommerce and WPML Language plugin, and build the ERP functionalities by ourselves with PHP, JS, and other web programming languages (see Fig. 3).

With customized plugin, the WordPress and WooCommerce combination could be also extended to sell items not yet in inventory using a custom-made production management feature not available in core WordPress or WooCommerce installation (Fig. 4).

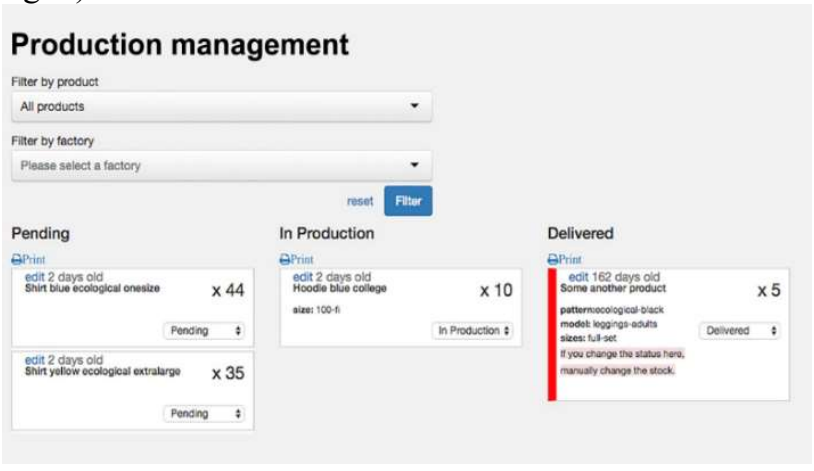

Fig. 4. Example of an ERP feature.
The production management feature showed real-time status of productions small business had to make: when the customer made an order

WooCommerce, order items were set in the production tracking to Pending category (see Fig. 4). When the

business started manufacturing the order, the business (administrator) changed the state 
to In Production followed by Delivered when the batch of ready products has been sent to the customers. When the business set the state to Delivered, the WooCommerce automatically sent the PDF invoice to the customer, based on contact information in CRM. This feature was very useful for businesses manufacturing and creating something by themselves allowing them to keep in track of their production progress.

As mobile-friendly usability was important in the system, responsive design was also designed to function at the interface of the production management system which could be easily used via mobile or different handheld devices as it automatically adopted the screen resolution. Custom-made features (like in Fig. 4) had to be visualized with CSS and JS coding, as PHP is mainly a server-side language. Customized PHP, JS, and PHP scripts formed a new WordPress plugin that could be installed in any WordPress using the platform's core plugin installation system (Fig. 3).

The installation steps getting the standalone WordPress platform running were straightforward. It only required a simple open source web-server server setup with Apache or Nginx with PHP and MySQL functions. Just in few minutes, the developer could have a WordPress system running with web-based administration capabilities in public web domain. After the installation, the business owner could install additional plugins needed for the system to tailor it for various business needs, such as ecommerce and ERP features.

However, as unsecured and non-professional WordPress installations could facilitate serious security threats to be used by the hackers, such as unauthenticated file uploads, object injections, and other exploits, instructions and suggestions given by the WordPress.org and its development community were rigorously followed.

The prototype WordPress installation got installed to the virtual server running inside one large dedicated server hardware (Fig. 5). This setup allowed to run multiple WordPress installations in one physical server. By using virtualization, each WordPress installation could be separated to own virtual servers for better security and reliability. In case one of the WordPress installations would get hacked, not all the installations would get compromised and the hardware server would stay safe. Usually this kind of setup is common for commercial cloud providers, such as ClearWeb Cloud Provider where the developer only rents the virtual server and pays for the resources used. The web-hosting providers maintains the hardware (dedicated) servers at their secure datacenters.

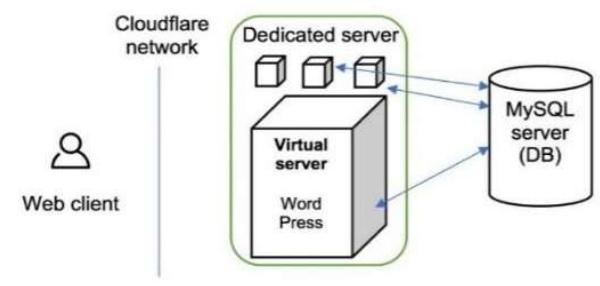

Fig. 5. Hosting WordPress platform in a web server.
The databases were separated from the virtual servers and held on a separate server running only MySQL databases of multiple systems (Fig. 5). The WordPress virtual servers were communicating with the databases using a private network in the same physical data center.

This arrangement of separate web and MySQL server was implemented because potential business growth could lead to higher page loads resulting increasing usage of the server resources. For example, some WordPress plugins made a significant load to the MySQL when a lot of various features were being used in a WordPress. To separate WordPress file system and MySQL, each installation could operate and grow on its 
virtual machine with limited resources not affecting the other systems.

In the prototype, additional security layer was achieved by using free Cloudflare.com service, which routed all the web traffic in and out from the WordPress web server. Cloudflare.com protected web applications and websites using a powerful and advanced framework processing the requests before they hit the server. For improved security, reliability, and performance in prototype, the traffic was routed through the Cloudflare.com network.

The evaluation of the constructed prototype was done according to guidelines of Hevner et al. [7] who emphasized the importance of a consistent and accurate assessment of the design process. The evaluation of the prototype took place in a simulated environment, where the features of the system were examined regarding functionality, usability, and efficiency. The evaluation was done manually by testing features through one by one with notes and also the evaluation data was automatically collected by using free Google Analytics platform that gathers data from browser sessions.

In the construction phase, the evaluation of the utilization and usability in the targeted context was done in a simulated environment, where all the features and functions were tested through. The evaluation included testing and observing users indirectly by letting them use the system and monitoring how they behaved in certain situations. It appeared that all the features of the system were functioning at satisfactory levels in desktop and mobile devices, which was the desired technical outcome of the prototype system.

In the evaluation phase, frontend usability of WordPress was tested. WordPress and many of its plugins supported mobile-friendly use, but extra work was required to improve it for satisfactory levels. Smartphone usability was considered important when developing systems for small businesses. As most of the small business users conduct and operate their businesses in smartphones, desktop level applications may cause issues for customers interacting with e-commerce platforms and ERP systems.

Google Analytics system was used to monitor how users browsed and interacted with the system: on backend using the features from product creation, website management to production and inventory management and CRM. The evaluation was followed by the frontend usability testing by adding products to shopping carts and monitoring how the customers checked out using selected payment methods.

By using the Google Analytics, it was possible to see which devices stayed on the ecommerce frontend the most and bounced the less when using the system. The iPad and Huawei users had the longest average session duration while the iPhones gave the most concerning results. Google Analytics was mainly monitored from the public frontend of the website to recognize the issues that e-commerce customers would face when trying to make purchases in the system. Usability on the frontend was determined more important than the usability on the backend because the income of the business owner was directly linked the customer satisfaction and sales coming from the public ecommerce section of the system.

The evaluation phase showed that it was important to keep the page loading time as small as possible and usability good as possible. In the simulation, the many pages were loading very slowly on mobile, resulting from a lot of unnecessary media (pictures, CSS, JS) what were loaded to the screen. Fast and mobile friendly site would lead to 
better customer satisfaction, longer session duration and finally increased sales which was suggested for further development.

\section{$4 \quad$ Discussion and Conclusions}

The motivation for the study was based on an existing small company that offered software services to its customers. The theoretical foundation was seen in the related literature, where e-commerce and ERP systems were understood to be part of larger enterprises meaning complex information systems. The purpose of the study was to build a prototype of a functional and cost-effective system to be used by small businesses when they grow their business by integrating open source e-commerce and EPR features. Cost-effectiveness was one of the most valued requirements (see [15]). The need for the solution was recognized in a Finnish software company ClearWeb. After the study, ClearWeb utilized the system in its service offering.

The first guideline in design science research is that a concrete artefact should be designed and developed [7]. This principle was achieved by designing and constructing the system. Also, evaluation should be done with recognized and valid metrics that fit the business environment where the product is designed [13]. The prototype evaluation was arranged in a simulated environment, where the features of the system were examined regarding functionality, usability, and efficiency. The data was collected from software-based evaluation using Google Analytics platform that gathered data from browser and server sessions. Evaluation using Google Analytics was determined sufficient, as it provided satisfactory and quick results without additional costs. Cost of employing the evaluation is crucial element in e-commerce evaluation [26]. Furthermore, the study produced new and innovative solutions that can be introduced in the operating environment of the product: after the study, the prototype was harnessed to a commercial software product in a real-world company ClearWeb.

In the study, we took the base of the free open source platform WordPress and started expanding it towards e-commerce and more advanced ERP features with available free plugins and custom PHP code. As WordPress was found open source software, it was possible to utilize and customize it only for the cost of development work. Absence of licensing expenses makes WordPress reasonable for small businesses to be connected respectively to the concept of small business growth.

Small businesses could use WordPress platform system from the start of its business (as a business website) well into its life with more advanced e-commerce and ERP platform [19]. The ERP system selection should support the nature of the business operations, not making it messy or hard to use [6]. In the evaluation phase, WordPress and its plugins were found usable and practical from the view point of small businesses.

All ERP features and success factor from larger enterprises are not practical to apply in small businesses as they can create complexity and other impractical technological challenges in small businesses [1]. ERP system hardware and software should be installed and mainly by the supplier's developers and technicians [6]. The current study showed that in WordPress, a skilled small business owner can do the installation and work without additional support requiring very basic level technical skills. Likewise, the WordPress system can be hosted in a virtual server in the cloud, without own server hardware. 
One of the most significant factors in e-commerce success and growth is the used infrastructure followed by reasonable costs of running and maintaining the system [15]. Based on the prototype of the current study, WordPress infrastructure seemed satisfactory for small business needs, requiring no other investment than time and interest as any other free open source software, as proposed by earlier knowledge [24]. However, some small server maintenance expenses were expected, but requirements for those were also very reasonable using Nginx or Apache open source server software and small server requirements.

Google Analytics suite has proven to be a valuable tool [13]. During our evaluation, it appeared an efficient tool for assessing usage of the system and to see which devices offered the best user satisfaction. The evaluation feedback from Google Analytics pointed attention to improve system to level accessible also via mobile devices, helping with integration into the daily lives of businesses using several mobile devices.

Future studies and efforts could be targeted to environments where users and companies are in their early stages of utilizing means of ICT to promote their economic development. Low-cost solutions such as WordPress-based platform can open fruitful international possibilities to small business.

\section{References}

1. Neirotti, P., Paolucci, E., Raguseo, E.: Is it all about size? Comparing organisational and environmental antecedents of IT assimilation in small and medium-sized enterprises. International Journal of Technology Management 11, 61(1), 82-108 (2013).

2. Kumar, R., Kumar Singh, R.: Coordination and responsiveness issues in SME supply chains: a review. Benchmarking: An International Journal, 24(3), 635-650 (2017). https://doi.org/10.1108/BIJ-03-2016-0041

3. Sabbagh, K., Friedrich, R.O.M.A.N., El-Darwiche, B.A.H.J.A.T., Singh, M.I.L.I.N.D., Koster, A.L.E.X.: Digitization for economic growth and job creation: Regional and industry perspective. The Global Information Technology Report, Chapter 1.2, 35-42 (2013).

4. Bansal, R.: Growth of the Electronic Commerce in China and India: A Comparative Study. Journal of Asia-pacific Business, 12(4), 356-374 (2011). https://doi.org/10.1080/10599231.2011.611457

5. Al-Ismail, M., Sajeev, A.: Usability challenges in mobile web. Retrieved September 30, 2017, from https://openresearch-repository.anu.edu.au/handle/1885/76586

6. Umble, E.J., Haft, R.R., Umble, M.: Enterprise resource planning: Implementation procedures and critical success factors. European Journal of Operational Research, 146(2), 241-257 (2003).

7. Hevner, A.R., March, S.T., Park, J., Ram, S.: Design science in information systems research. Management Information Systems Quarterly, 28(1), 75-105 (2004).

8. Greiner, L.E.: (1972). Evolution and revolution as organizations grow. Reprint. Harvard Business Review May-June 1998, 3-11.

9. Carneiro, A.: "What is required for growth?", Business Strategy Series, Vol. 8 Issue: 1, pp.51-57 (2007), https://doi.org/10.1108/17515630710686888

10. Roig-Tierno, N., Alcazar, J., Ribeiro-Navarrete, S.: Use of infrastructures to support innovative entrepreneurship and business growth. Journal of Business Research, 68(11), 2290-2294 (2015). https://doi.org/10.1016/j.jbusres.2015.06.013

11. Kenney, M., Rouvinen, P., Zysman, J.: The Digital Disruption and its Societal Impacts. Journal of Industry, Competition and Trade, 15(1), 1-4 (2015). 
12. Wang, Y.: Achieving market responsiveness: The enabling role of it and e-commerce adoption in SMEs. In International Conference on Management and Service Science (MASS), 2010 (pp. 1-4). IEEE. DOI: 10.1109/ICMSS.2010.5576558

13. Burzacca P., Paternò F.: Remote usability evaluation of mobile web applications. In: Kurosu M. (eds) Human-Computer Interaction. Human-Centred Design Approaches, Methods, Tools, and Environments. HCI 2013. Lecture Notes in Computer Science, vol 8004. Springer, Berlin, Heidelberg (2013).

14. Boateng, R., Hinson, R., Heeks, R., Molla, A.: E-commerce in least developing countries: Summary evidence and implications. Journal of African Business, 9(2), 257-285 (2008).

15. Choshin, M., Ghaffari, A.: An investigation of the impact of effective factors on the success of e-commerce in small- and medium-sized companies. Computers in Human Behavior 66, 67-74 (2016).

16. Fitzgerald, M., Kruschwitz, N., Bonnet, D., Welch, M.: Embracing digital technology: A new strategic imperative. MIT Sloan Management Review, 55(2), 1-12 (2014).

17. Lichtenstein, G A., Lyons, T.S.: Revisiting the business life-cycle: proposing an actionable model for assessing and fostering entrepreneurship. The International Journal of Entrepreneurship and Innovation, 9(4), 241-250 (2008).

18. Baack, S.: Datafication and empowerment: How the open data movement re-articulates notions of democracy, participation, and journalism. Big Data \& Society, 2(2) (2015) 2053951715594634.

19. Woocommerce.com.: WooCommerce. Retrieved May 15, 2018 from: https://woocommerce.com/

20. WordPress.org.: WordPress. Retrieved May 18, 2018 from WordPress: https://wordpress.org

21. BuiltWith.: Ecommerce Usage Statistics. Retrieved September 15, 2017, from BuiltWith Web Technology Usage: https://trends.builtwith.com/shop

22. Perez-Estebanez, R., Urquía-Grande, E., Rautiainen, A.: Technological and economic factors determining ICT level: Evidence from rural micro businesses in Democratic Republic of Congo. Journal of International Development, 30(1), 118-133 (2018).

23. EC.: http://ec.europa.eu/growth/smes/business-friendly-environment/sme-definitio_en Retrieved September 20, 2018.

24. Carland J.W., Hoy F., Boulton W.R., Carland J.A.C.: Differentiating entrepreneurs from small business owners: A conceptualization. In: Cuervo Á., Ribeiro D., Roig S. (eds) Entrepreneurship. Springer, Berlin, Heidelberg, pp. 73-81 (2007).

25. Dutta, P., Ahmed, S.: Review of literature of determinants of ERP implementation in SMEs. Journal of Basic and Applied Engineering Research 3(12), 1100-1105 (2016).

26. Hasan, L., Morris, A., Probets, S. G.: Using Google Analytics to evaluate the usability of E$\begin{array}{llllll}\text { Commerce } & \text { sites. } & \text { Retrieved } & 10 & 2618, & \text { from }\end{array}$ https://link.springer.com/chapter/10.1007/978-3-642-02806-9 81 